\title{
A COMMUNICATION MANIFESTO (EVOLVING)
}

\author{
STANISŁAW PUPPEL
}

1. Communication is fundamental in our world. It means that communication is both a basic human need and a fundamental social process by means of which human society becomes integrated. According to The Declaration of Principles, prepared by the World Summit on the Information Society (WSIS) in Geneva (2003), "We are firmly convinced that we are collectively entering a new era of enormous potential, that of the Information Society and expanded human communication. In this emerging society, information and knowledge can be produced, exchanged, shared and communicated through all the networks of the world. All individuals can soon, if we take the necessary actions, together build a new Information Society based on shared knowledge and founded on global solidarity and a better mutual understanding between peoples and nations. We trust that these measures will open the way to the future development of a true knowledge society" (Principle 67). The following points of the Communication Manifesto demonstrate the nature of the common foundation upon which all human communication rests.

2. Communication is embodiment-based. It means that communication is possible between/among living entities (i.e. bodies) generated by the conditions of the Earth (i.e. the world) as the vehicle of all life. In this perspective, the world is a source of inputs to the body and an arena for outputs produced by the body (i.e. actions effected by the body).

3. Communication is sharing-based. It means that communication is an individual act of sharing of the various resources possessed by a living organism, including the human communicating agents (hence HCA, also the human communicators, or simply 'communicators', Homo communicans) but also including 
the human-constructed machines communicating with other participants of the act. The possible combinations of sharing include: plant - plant, plant - animal, animal - animal, human - plant, human - animal, human - human, animal machine, human - machine, machine - machine.

4. Communication is cohabitation-based. It means that, overall, the various communication designs encountered in Nature as the most general habitat tend to complete each other (i.e. they coexist with each other and stay in some form of symbiosis both across the species and/or within one particular species) thus forming a Universal Cohabitation Design. Within the Design, the following patterns of cohabitation may be found: mutualism, commensalism, amensalism, and parasitism. Consequently, one may postulate that all the HCAs form the Communicative Cohabitation Design. Therefore, in communicative practice the following types of cohabitation may be found: communicative mutualism, communicative commensalism, communicative amensalism, and communicative parasitism, respectively.

Communicative mutualism may be defined as the kind of cohabitation where each interacting HCA is able to derive communicative fitness or improved communicative efficiency from communicative interactions. This type of interaction may also be termed 'cooperation'.

Communicative commensalism may be defined as the kind of cohabitation where one communicator is able to obtain benefits while the other communicator is neither benefited nor harmed.

Communicative amensalism may be defined as the kind of cohabitation where during communicative interaction one communicator is harmed (i.e. communicative behavior is detrimental to him/her) whereas the other is neither affected nor benefited in any way.

Communicative parasitism may be defined as the kind of cohabitation where one communicator (defined as the 'parasite') benefits at the expense of the other communicator (defined as the 'host').

5. Communication is interactivity-based. It means that the communicators are able to participate in the process of generating and modifying the structure and content of communicator-mediated environment in real time. In addition, the communicators are capable of acting on each other, on the communication me$\operatorname{dium}(\mathrm{s})$, and on the messages such that these impacts are synchronized (socalled synchronicity/synergy in interaction) and actively controlled.

6. Communication is transmission-based. It means that, apart from being an act of sharing, communication is also regarded as 'transmission', where the following basic elements can be distinguished: an information source (sender), a channel of transmission (e.g. the audio-vocal channel, the visual-tactile chan- 
nel, or a combination of channels), a destination (a receiver), a code, and noise which is a dysfunctional element always present in the process of transmission and for ever interfering in the communication acts.

7. Communication is resource-based. It means that the HCAs communicate with each other within the confines of their cultural (ethnic), linguistic (i.e. verbal) non-linguistic (i.e. non-verbal), and psychological (e.g. cognitiveemotional) resources accrued by and available to the HCAs. The whole complex of culture-language-psychology is responsible for the generation of communicative diversity by the particular HCAs in countless many communication acts.

8. Communication is management based. It means that in manufacturing the various messages we are involved in tailoring them to the demands of the potential and imminent recipients. That is, we are involved in the generation of various communicative strategies whereby we both link our messages with the linguistic and non-linguistic resources which we own and which act as resource constraints, and further use them to express our intentions to the recipients. Management-based communications are part and parcel of a gigantic process of collective bargaining which takes place in the open public sphere.

9. Communication is channel-based. It means that in the domain of human communication, communication acts are delivered in the following modalities: the audio-vocal modality (AVO), the visual-tactile modality (VIT), and the mixture of both, the hybrid modality (HYB).

10. The AVO modality keeps the HCAs in the primary communication order, the oral/vocal order of communication (Homo loquens).

11. The VIT modality transfers the HCA into the secondary communication order, the written/graphic order of communication (Homo scribens).

12. The HYB order of communication, in turn, is the most blended communication order, the most expandable, most inclusive and technologically the most advanced order of communication.

13. Communication is sender-based. It means that the communicator, in order to be received and perceived, must produce a message which requires performance in the more or less open public space.

14. Communication is performance-based. It means that the particular HCAs, as they are involved in communication acts, are also involved in the process of message preparations which comprises the planning and engineering of resource use (i.e. which performance channel, to what extent, with what quality, how much synergy across the channels, and in what emotional layout), resource activation and integration in a particular communication act which involves the 
entire implementation and manufacturing complex. Thus, communicator involvement is crucial for the performance-based communicative behavior.

15. Communication is receiver-based. It means that the communicator is more or less aware of the entire implementation complex, that is, $\mathrm{s} / \mathrm{he}$ is performing in the social milieu, whereby the receiver, both/either an individual receiver and/or a group of receivers (receiver identification), are addressed.

16. Communication is effect-based. It means that the communicator wishes to attain a more or less clearly identified communicative goal/task (i.e. there is a sense of purpose, communicative mission) while activating and integrating his/her resources in the act of communication. The tasks, among others, may include: information exchanges, as well as specialized communicative behaviours such as moderations/mediations, negotiations, persuasions, coercions, also language and non-language displays for gaining mere aesthetic pleasure.

17. Communication is accountability-based. It means that the HCA as the sole owner of his/her resources is kept accountable for his/her performances. The communicative successes and failures of any HCA are the result of the communicator's more or less conscious choices made in the course of the communication act as well as of the communicator's overall organizational capacity (organizational robustness). Accountability is expressed by means of the following parameters: accountability is understood as the relationship of the communicators in the sender-receiver dyad, accountability is goal-oriented, accountability is meaningless without individual and social consequences of the communication acts, accountability requires various forms of feedback, accountability leads to overall improvements in the communicator's performance potential. Accountability has the following dimensions: personal accountability (i.e. an accountability of the communicator's relationship with oneself), social accountability (an accountability relationship with the other communicators as well as with groups of communicators).

18. Communication is auto feedback-based. It means that the communicator, while sending the messages out to the receiving end, is also able to (self)monitor his/her communicative behavior. In this way, corrections may be introduced at any point of the communication act.

19. Communication is code-based. It means that the communicator has at his/her disposal a specialized code, or a set of codes, which may be selected and activated in various communication acts. The code comprises a limited number of structural-functional (discrete) units which may be assembled and reassembled in an infinite number of meaningful ways (i.e. replicability of units, the discoursive and recursive power of the code) while, at the same time, adhering to and demonstrating the structural stability of the variously sized discrete units. 
20. Communication is replication/creativity-based. It means that, as has been stated above, the communicator has at his/her disposal a limited number of discrete units which, on the one hand, limit (i.e. quantify) the communicator's resources and, on the other, may be used in a variety of ways, also including innovations, to meet the demands of the constantly changing context. In this way, all the communicators are creative communication activists and socially endorsed creators of messages. Simply, the presence of creativity in communication, based on the replicability of the codes, is an essential part of language-generated human culture.

21. Communication is environment-based. It means that in order for a communication act to be accomplished in a proper way, the communicator must be aware of the environmental ramifications of any communication act and be able to include them as the context of communication. Simply, the communicator is required to perform constant environmental scanning both for his/her individual safety and for the necessity of updating the overall 'positioning' of the individual HCA within a broader social-cultural communicative context (i.e. in any human habitat, e.g. the city as a node of communication or any other communication arena).

22. Communication is immersed in noise. It means that noise is a permanent component of any communication act. Depending on its volume, the presence of the noise component may cause greater or smaller interferences in the communication process by leading to more or less serious distortions of the content and during the transmission of the messages.

23. Communication is closure-based. It means that the communicators are aware of the temporal, spatial, and logico-semantic limitations of any communication act. The latter is simply incomplete without applying an effective closure which is the final step in this act, both psychologically and implementationally.

24. Communication is institution-based. It means that it takes place in a complex social (hierarchic and heterarchic) and institutional setup where it may run multidirectionally. That is, it may run from top to the bottom of the institution (the top-down communication), from the bottom to the top (bottomup communication), and horizontally (in heterarchic, democratic, and ecocratic forms of communication).

25. Communication is individual-based. It means that the individual communicators are involved in the various communication acts in which the communicative resources are implemented with the intention of attaining individual goals. In this framework, communication outputs are intended to serve, influence or target the individual communicators. 
26. Communication is collaboration-based. It means that the individual communicators are involved in a collaborative communication process in which the various communication acts are implemented with the intention of attaining a collaborative goal as well as various public goals (Homo communis, Homo publicus). In this framework, communication outputs are intended to serve, influence or target larger groups of people (i.e. institutions and mass communication) in the open public space.

27. Communication is network sensitive. It means that all the communicators may (and do) participate in social networks as collectivities of communicators functioning as 'nodes' of communication with multiple interlikages, both real and virtual. The power of social network performativity is much larger than the power of the performativity of individual communicators. Moreover, the individual communicators' affiliations in the networks involve the qualityquantity dimension of the cultural and linguistic resources which are shared by the communicators. Thus, their very use serves to maintain and strengthen the resources of a given natural language as well as it serves to maintain and strengthen the identity of the individual communicators as nodes in the network (Homo reticulosus).

28. Communication is focus-based. It means that in its implementation process, the content is more or less focused on ever changing communicative needs of the human communicating agents. It is relevant.

29. Communication is flexibility-based. It means that the communicators take full advantage of their resources (both qualitatively and quantitatively) and constantly alter their communicative performances thus moving freely between and across the communicative niches and the linguistic resources (i.e. their size and quality) which serve to identify them (Homo fluxus).

30. Communication is niche-based. It means that the communicators implement their resources basically within the following three fundamental communicative niches: the daily routine and general culture niche, the professional niche, and the citizenship niche. Each of the niches may be characterized by its own type (i.e. size, frequency and quality) of linguistic resources. Subsequently, the daily routine and general culture niche, the largest in size and most frequently used, is the one which comprises the most fundamental (and most sizable) linguistic resources thus allowing the communicators to communicate on matters of daily life. The professional niche, smaller in size and less frequently used, is the one which comprises the most sophisticated professional jargon and most technical vocabulary tailored by the particular professional bends. Finally, the citizenship niche, smallest in size and least frequently used, is the one which com- 
prises all the sophisticated linguistic resources of the legal ramifications of the communicators' existence within society.

31. Communication is niche-alignment-based. It means that every communicator uses a uniquely aligned (i.e. highly individualized) and highly variable repertoire of communicative devices derived from the size and quality of his/her resources and implements them in a particular communication act. The alignment of the outcome requires that the particular niche resources be properly controlled and synchronized by the communicators.

32. Communication is culture-based. It means that every communicator is rooted in culture as the ever comprising institutional environment, and in a particular local (i.e. ethnic) culture which is an instantiation of culture as an ever present dimension of the human predicament. With respect to culture as such, the human communicators are defined identity-wise as generally conforming in their communicative behaviours to the interplay of the following parameters: 'militancy' (i.e. a certain degree of combativeness/aggressiveness demonstrated towards other HCAs), 'trade-offs' (i.e. a certain degree of readiness of a HCA to apply operational routine compromises towards other HCAs such that information exchanges are secured for each communicator), 'utility' (i.e. the communicators get involved in the communication acts along the 'resource as a goodservice' dimension: in terms of the resource regarded as a good, the particular HCAs are engaged in lifelong accretion of cultural, linguistic and non-linguistic resources as both the intangible assets which characterize the human species and as the potential sources of whatever utility for the future communicative behaviours, whereas service-wise, the HCAa are involved in the kind of culturallinguistic-communicative conduct which is positively valued by society), and 'display' (i.e. the HCAs are necessarily functioning in a multi-display environment such that they are involved in signaling to each other their overall integrity, efficiency potential, overall attractiveness, sense of aesthetics, and their readiness for interactions and communication).

33. Communication is 'stakeholder'-based. It means that every communicator is a stakeholder, that is, $\mathrm{s} /$ he is both affected (or impacted) by the outcomes of a particular communication act and affects (or makes an impact on) other communicators. In this way, the communication process is also biased by its economy, that is, it is regarded as approached within the economic framework of the costs and gains (benefits) of communication understood as an act of sharing (Homo oeconomicus).

34. Communication is cost-based. It means that every communicator is aware of the problem of cost involvement in the manufacture of an act of communication. Simply, every communicator is involved in the process of estima- 
tion as to how much communicative surplus (as part of the resources owned by the individual HCA) $\mathrm{s} / \mathrm{he}$ is willing to invest in a particular communication act. This is the problem of quantity in communication.

35. Communication is benefit-based. It means that every communicator expects communication benefits, either direct or indirect, resulting from an involvement in various communicative exchanges. The direct benefits may comprise the following: direct information gains, aesthetic (hedonic) gains, intellectual and emotional gains, material gains. Whereas, the indirect benefits may include: prospective gains in long-term seeking of information, long-term intellectual/emotional gains, long-term material gains, long-term aesthetic gains, etc. This is the problem of the quality of communication.

36. Communication is information-based. It means that the communicative exchanges which take place between/among the communicators have one major purpose: to exchange information understood as a sequence symbols assembled into a message (or a set of messages). In this sense, communication is: coded, constrained, linear, manageable, controlled, mediated, meaningful, significant, data-oriented, credible, comprehensible (also commonsense), structured, representational, directed and demonstrating negentropy (i.e. it is non-chaotic).

37. Communication is error prone. It means that communication is permeated by all kinds of errors and slips. Subsequently, every communicator is most naturally prone to commit all kinds of errors while performing various acts of communication. Errors may pervade the entire structure of language, the pragmatics of verbal and non-verbal resource usage as well as all the stages of the very process of the manufacturing of the messages.

38. Communication is learning-based. It means that all communication in embodied HCAs is determined by antecedents which are, generally, the whole 'body' (i.e. repertoire) of stimuli that get the communicative behavior started. The antecedents, which supply information concerning how successful a previous communication act was, include the stationarity of the world as the vehicle for all living agents (the so-called situatedness, or environmentally conditioned existence of the communicators who act through the constraints of the body), the complexity of the situatedness, identified errors and slips which have occurred in previous communicative behavior. Properly applied antecedents, that is, effected through a synergistically functioning and intricate system of cognitive controllers, lead to important consequences, namely to the establishment of a better control of communicative behavior and thus enable and facilitate efficient learning such that the communicators are able to introduce improvements in their communicative behaviours in order to communicate effectively, successfully, 
and comfortably. In this way, the communicators can finally attain a better standard of 'communicative wellbeing' in the communication arena.

39. Communication is sustainability-based. It means that every single communication act in which a particular HCA participates and which involves the activation of the linguistic and non-linguistic resources and of the medium of communicative performance, contributes to (or has a positive impact on) the preservation of these resources and of the media. In this way, communication is of utmost ecological significance.

40. Communication is diversity based. It means that while building the future Information Society we must demonstrate particular care and respect for different cultural identities, diverse languages and traditions. If possible, we must also participate in the promotion and preservation of all the existing cultural identities and natural languages, both on a global and local (i.e. domestic and regional) scale.

41. Communication is design-based. It means that human communication takes place in a larger structure, the Human Communication Design. The Design comprises the following: the Earth as the vehicle and seat of input/output activities in the sense defined in (2) above, the human species as a population of active and potential communicators, the resources (among them: the abiotic and biotic ones, human, psychological, social, cultural, linguistic, non-linguistic), as well as the communicative niches. The design, in turn, is part of the ever encompassing Universal Communication Space where all the other communication designs can be encountered (e.g. the plant communication design and the animal communication design) and where they can encounter each other. The communicator who is able to communicate successfully and comfortably across the niches, across local cultures and local languages is termed the transcommunicator.

42. Communicamus ergo sumus! This invocation illustrates the ultimate fate of the human beings realized via the communicative potential and diversified communicative behaviours as indicated in the opening point (1) of the Communication Manifesto. Its presence and realization equals the ontic anchoring of every human being in the communicative milieu as the basic social-cultural milieu. 
